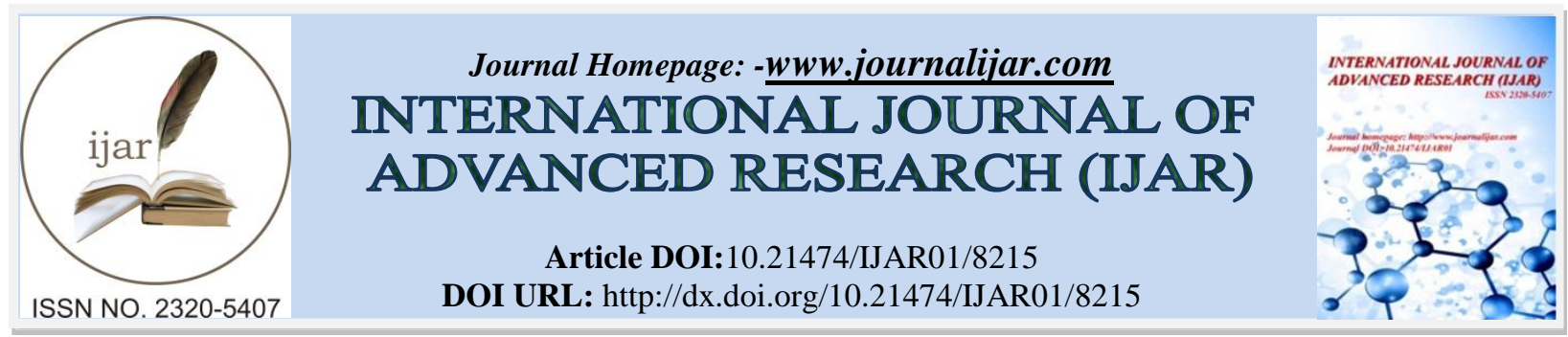

RESEARCH ARTICLE

\title{
LES ATTEINTES OCULOMOTRICES AU COURS DU DIABÈTE.
}

\author{
H. Moata ${ }^{1}$, G. El mghari ${ }^{2}$ and N.el Ansari ${ }^{2}$. \\ Service d'endocrinologie, diabetologie, maladies metaboliques et nutrition laboratoire pcim, fmpm. Universite \\ cadi ayad. Chu mohamed vi. Marrakech.
}

\section{Manuscript Info}

Manuscript History

Received: 16 October 2018

Final Accepted: 18 November 2018

Published: December 2018

\begin{abstract}
Le but de notre travail est de souligner l'importance de l'équilibre glycémique, et de la correction des facteurs de risque vasculaires dans le traitement et la prévention des paralysies oculomotrices au cours du diabète (POM) ainsi que l'intérêt de l'examen de la motilité oculaire chez tout sujet diabétique et la nécessité d'un bilan étiologique devant toute POM diabétique afin d'écarter une urgence neurochirurgicale .

Matériel et méthodes : Nous rapportons une série de 7 patients diabétiques, atteints de paralysie oculomotrice, colligés au service d'endocrinologie - diabétologie du CHU Med VI de Marrakech entre 2013 et 2018.

Résultats : Notre étude a porté sur 2 hommes et 5 femmes. L'âge moyen était de 49 ans et 8 mois. Le diabète était de type I dans 1 cas et de type II dans 6 cas. L'examen de la motilité oculaire a objectivé une paralysie du VI unilatérale dans 2 cas, une paralysie du IV dans 3 cas et une paralysie du III dans 6 cas . La tomodensitométrie cérébrale s'est révélée normale dans tout les cas. L'évolution s'est faite vers la récupération progressive mais pas totale de la paralysie après une moyenne de 5 mois de traitement par occlusion alternée.

Conclusion : L'installation de la paralysie oculomotrice diabétique est relativement lente et peux être précédée de douleurs oculaires, périorbitaires ou même hémicrâniennes homolatérales. Son évolution est favorable en quelques semaines ou mois, mais la récidive du même côté ou du côté controlatéral est possible.
\end{abstract}

Copy Right, IJAR, 2018,. All rights reserved.

\section{Introduction:-}

Les paralysies oculomotrices au cours du diabète (POM) sont des mononeuropathies focales. Il s'agit d'une complication neurologique diabétique, relativement rare puisque l'incidence selon les auteurs est de 1 à $14 \%$ chez les sujets diabétiques. Elle est 7 à 8 fois plus fréquente chez les sujets diabétiques que chez les non diabétiques [1].

Les atteintes oculomotrices, parfois premiers signes d'un diabète de type II, représentent pour le patient un événement souvent brutal, aux conséquences immédiates très handicapantes .Les nerfs oculomoteurs touchés sont essentiellement le nerf oculomoteur externe (VI), le nerf oculomoteur commun (III), et plus rarement, le nerf pathétique (IV).

Corresponding Author:-H. Moata.

Address:-Service d'Endocrinologie, Diabétologie, Maladies métaboliques et Nutrition. 
A travers 7 cas de paralysie oculomotrice chez des sujets diabétiques, nous allons étudié les particularités cliniques, évalué les facteurs de risque et étiopathogéniques et l'apport de l'imagerie cérébrale pour le diagnostic différentiel, ainsi que la prise en charge thérapeutique et le suivi évolutif.

\section{Matériel Et Méthodes:-}

Notre étude porte sur 7 cas de paralysies oculomotrices chez des patients diabétiques, colligés au service d'endocrinologie - diabétologie du CHU Med VI de Marrakech entre 2013 et 2018.

À l'interrogatoire, nous avons pu préciser le type de diabète et la durée d'évolution ; apprécier la qualité d'équilibre glycémique ; noter l'existence d'une hypertension artérielle, d'une rétinopathie diabétique et quantifier la durée d'évolution de la paralysie oculomotrice.

Tous ces patients ont bénéficié d'un examen ophtalmologique avec examen dynamique de la motilité oculaire et des ductions (abduction et adduction) .Un Examen général a la recherche d'autres complications diabétiques, ainsi qu'un examen neurologique complet a été réalisé a la recherche d' atteinte des autres paires crâniennes, d'une neuropathie sensitivomotrice distale, d'un syndrome cérébelleux...

L'examen ophtalmologique a été complété par :

1. un bilan du diabète (Hémoglobine glycosylée ; évaluation de la fonction rénale ; évaluation cardiaque et des facteurs de risque cardiovasculaire : bilan lipidique et électrocardiogramme)

2. bilan orienté selon la clinique permettant d'éliminer d'autres diagnostics étiologiques de la POM: notamment les Sérologies (syphilis, HIV, tuberculose) et l'imagerie cérébrale.

\section{Résultats:-}

1. Notre série porte sur 7 patients, 2 de sexe masculin, 5 de sexe féminin. L'âge moyen est de 49 ans et 8 mois, avec des extrêmes de 21 et 70 ans.

2. Le diabète est de type I pour 1 patient et de type II pour 6 patients. L'ancienneté du diabète est en moyenne de 12 ans, avec des extrêmes de 5 à 20 ans. Ce dernier était mal suivi dans 3 cas et non suivi dans 2 cas et inconnu dans 2 cas .

3. Le statut dégénératif est inconnu chez 6 patients, une rétinopathie diabétique était retrouvé chez une seul patiente traité par photocoagulation .

4. 1 patient était sous antidiabétiques oraux, insulinothérapie chez 5 patients et 1 patient était sous traitement mixte.

5. L'hypertension artérielle et l'hypercholestérolémie révélée par le bilan lipidique ont été notées chez presque la moitié des patients.

6. La paralysie oculomotrice était d'apparition subite et en concomitance avec une décompensation cétosique dans 3 cas, pour les autres patients la POM était d'apparition et d'aggravation progressive avec une consultation motivée par une diplopie gênante pour ces patients. Cette diplopie était associée à des céphalées dans 2 cas .

7. L'examen de la motilité oculaire a objectivé une paralysie du VI unilatérale dans 2 cas, une paralysie du IV dans 3 cas et une paralysie du III dans 6 cas .

8. L'examen ophtalmologique n'a révélé une rétinopathie diabétique que dans 1 cas et la tomodensitométrie cérébrale s'est révélée normale dans tout les cas .

9. Tout les patients avaient un déséquilibre glycémique, la moyenne d'Hbalc était de $10,2 \%$. La prise en charge endocrinologique a consisté en une insulinothérapie intensifiée pour tous les patients.

10. L'évolution s'est faite vers La récupération progressive mais pas totale de la paralysie après une moyenne de 5 mois de traitement par occlusion alternée.

Tableau I:-Caractéristiques cliniques des patients

\begin{tabular}{|r|r|r|r|r|r|r|r|}
\hline CAS & AGE & SEXE & $\begin{array}{r}\text { TYPE DE } \\
\text { DIABETE }\end{array}$ & $\begin{array}{r}\text { ANCIENNETE } \\
\text { DU DIABETE }\end{array}$ & $\begin{array}{r}\text { STATUT } \\
\text { DEGENERATIF }\end{array}$ & HTA & TRAITEMENT \\
A \\
\hline 1
\end{tabular}




\begin{tabular}{|r|r|r|r|r|r|r|r|}
\hline 3 & 58 ans & $\mathrm{F}$ & DT2 & inaugurale & Non \\
& & & & inconnu & + \\
connu \\
\end{tabular}

Tableau II:-Anomalies de l'examen des paires crâniennes

\begin{tabular}{|c|c|c|c|c|c|c|c|}
\hline CAS & AGE & SEXE & $\begin{array}{l}\text { TYPE DE } \\
\text { DIABETE }\end{array}$ & $\begin{array}{l}\text { ANCIENNETE } \\
\text { DU DIABETE }\end{array}$ & $\begin{array}{r}\text { STATUT } \\
\text { DEGENERATIF }\end{array}$ & HTA & $\begin{array}{r}\text { TRAITEMENT } \\
\text { A } \\
\text { L'ADMISSION }\end{array}$ \\
\hline 1 & 70ans & $\mathrm{M}$ & $\mathrm{DT} 2$ & 5ans & inconnu & + & Insuline+ADO \\
\hline 2 & 21 ans & $\mathrm{F}$ & DT1 & $\begin{array}{r}\text { Cétose } \\
\text { inaugurale }\end{array}$ & inconnu & - & - \\
\hline 3 & 58ans & $\mathrm{F}$ & DT2 & $\begin{array}{r}\text { Non } \\
\text { connu } \\
\text { diabétique }\end{array}$ & inconnu & + & - \\
\hline 4 & 62ans & $\mathrm{F}$ & DT2 & 20ans & inconnu & + & Insuline \\
\hline 5 & 64ans & $\mathrm{F}$ & $\mathrm{DT} 2$ & 15 ans & $\begin{array}{r}\text { Rétinopathie } \\
\text { diabétique }\end{array}$ & + & $\mathrm{ADO}$ \\
\hline 6 & 56ans & $\mathrm{F}$ & $\mathrm{DT} 2$ & 9ans & inconnu & - & Insuline \\
\hline 7 & 55 ans & $\mathrm{M}$ & DT2 & 11 ans & connu & - & Insuline \\
\hline
\end{tabular}

Figure I:-ptosis unilatéral

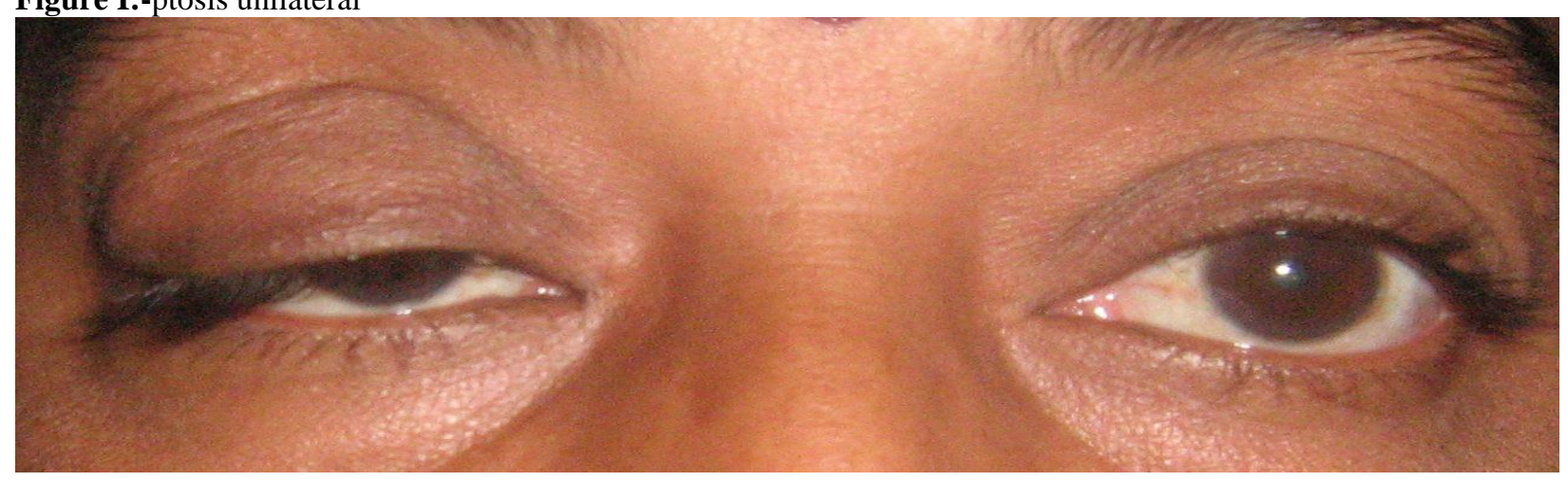

\section{Discussion:-}

De 2013 à 2018, seuls 7 cas de paralysie oculomotrice (POM) chez des patients diabétiques ont été colligés dans notre service. Ce chiffre est probablement sous évalué vue l'importance du recrutement de patients diabétiques, en 
effet l'absence d'examen minutieux et systématique de la motilité oculaire, fait que certaines parésies peuvent passer inaperçues, si elles ne sont pas révélées par une diplopie gênante.

Les POM surviennent presque toujours après 50 ans. Des cas sont néanmoins notés chez les sujets jeunes [2]. L'âge moyen du début de la paralysie dans notre série (49 ans et 8 mois) semble correspondre aux données de nombreux auteurs. Pourtant, il ne s'agit pas d'une pathologie du sujet âgé puisque nous avons recruté des sujets jeunes également (21 ans) [3] [4]

Il n'existe pas de corrélation entre la survenue de POM et le sexe. La POM intéresse aussi bien les patients diabétiques de type 1 que de type 2 [5].

La prévalence de la POM augmente avec la durée du diabète et le déséquilibre glycémique dans certaines études. Alors que dans d'autres études il n'existe pas de corrélation entre la durée et l'intensité du diabète et la survenue de POM. [6].

L'ancienneté du diabète (moyenne de 12 ans chez nos patients), le mauvais équilibre glycémique (chez tout les patients) sont des facteurs de risque significatifs de survenue des POM, mais ne sont pas suffisants pour expliquer à eux seuls ces paralysies. Les POM peuvent en effet être révélatrices d'un diabète dans 30 à $50 \%$ des cas, et survenir chez des patients avec bon équilibre glycémique.

Ainsi dans la série de Lazzaroni, portant sur 44 patients, 19 patients ont une POM révélatrice du diabète de type 2, alors que 25 patients ont un diabète de type 2 déjà connu avec une moyenne diagnostique de 9,5 6,2 ans. La qualité du contrôle glycémique est également déterminante dans l'apparition de la POM [7].

Nos observations rejoignent également les données de la littérature sur divers points. Dans notre série, comme dans celles de Goldstein et Cogan (1960), de Green etcoll. (1964), de Lazzaroni et coll. (1993) [8]

1. les troisièmes nerfs crâniens sont les plus fréquemment atteints.

2. La proportion de diabète de type II est plus importante (6 cas sur 7).

3. Nous ne relevons aucune relation entre la survenue de la POM et l'existence d'une rétinopathie diabétique (1 cas seulement dans notre série).

L'origine vasculaire étant incriminée dans la pathogénèse de ces POM, nous avons recherché chez nos patients des facteurs de risque vasculaire autres que le diabète, il s'agit d'une HTA avec hypercholestérolémie chez la moitié des patients

Par ailleurs, le pourcentage élevé de maladies vasculaires en général chez le diabétique, suggère le rôle d'un facteur vasculaire dans l'apparition des POM chez le diabétique. [9], [10].

L'ischémie semble être le principal facteur incriminé dans la survenue des neuropathies focales et multifocales d'origine diabétique en général et des POM en particulier. Dreyfus et al[11]., sont les premiers à soulever l'hypothèse d'une origine ischémique à cette manifestation. . [12], [13].

Sur le plan clinique, l'installation de la paralysie était associé a des céphalées dans deux cas, de tels phénomènes sont notés chez 50\% des patients [14]. Ils sont à l'origine d'une grande anxiété. Ces céphalées disparaissent assez vite. Leur intensité est généralement modérée, mais elle peut être intense sans exclure pour autant le diagnostic [15]., Dans la série de 44 patients de Lazzaroni, il ne semble pas y avoir de corrélation entre la POM et la fonction rénale, la présence de rétinopathie diabétique et les autres complications dégénératives diabétiques. [16].,

Selon un bon nombre d'auteurs, le diagnostic étiologique des POM chez le diabétique passe nécessairement par l'IRM cérébrale qui met en évidence l'atteinte des noyaux oculomoteurs, alors que la TDM peut être normale ou montrer uniquement des petits foyers hémorragiques [17], [18].

L'imagerie cérébrale (TDM, IRM) permet également d'éliminer les autres étiologies des POM notamment une compression tumorale ou anévrysmale ... 
Sur le plan thérapeutique, dans la mesure où les complications du diabète sont en grande partie une conséquence de l'hyperglycémie prolongée, il est logique d'essayer d'obtenir le meilleur contrôle glycémique possible. Le plus souvent lorsque l'équilibre glycémique n'est pas atteint sous antidiabétiques oraux, le recours à l'insulinothérapie s'avère nécessaire. Il ne faut pas oublier le traitement des autres facteurs de risque d'ischémie (Dyslipidémie, hypertension artérielle..) [19]

L'évolution reste souvent favorable en quelques mois, comme c'est le cas pour nos patients, ce qui suppose une origine vasculaire à ces POM. Jacobson et Broste, lors d'une étude comparative ont montré que plus l'installation de la paralysie était brutale, plus la résolution était rapide ce qui permet d'émettre un pronostic face à ce type d'atteinte . Néanmoins des récidives du même côté ou du côté opposé restent possibles, voire la survenue de paralysie des autres paires crâniennes, ce qui impose une surveillance ultérieure.

\section{Conclusion:-}

1. Les Paralysies oculomotrices sont relativement rares au cours de l'évolution du diabète.

2. Elles sont caractérisées par une apparition brutale, douloureuse de la diplopie et une évolution parfois spontanée vers la rémission.

3. Elles relèvent le plus souvent d'un mécanisme vasculaire ischémique et doivent être recherchées systématiquement chez tout diabétique par une étude minutieuse de la motilité oculaire.

4. La confirmation de l'origine diabétique d'une POM demeure un défi pour le clinicien. Certaines situations sont très confuses vue le polymorphisme clinique. La hantise est de méconnaitre certaines urgences diagnostiques, telle une cause tumorale, un anévrysme, une maladie de Horton ...... ce qui impose une réflexion profonde et précise ainsi q'une bonne exploitation des données cliniques.

\section{References:-}

1. Vighetto A, Tilikete C. Paralysies oculomotrices douloureuses : Une approche

2. diagnostique. Rev Neurol $2005 ; 161: 531-542$.

3. Menerath JM, Kantelip B. Manifestations ophtalmologiques du diabète (à l'exception de la rétinopathie diabétique et de la cataracte). Éditions Techniques, Encycl Méd Chir (Paris-France), Ophtalmologie, 21452D10, 1994,9 p.

4. Fujioka T, Segawa F, Ogawa K, Kurikara T, Kinoshita M. Ischemic and hemorrhagic brain stem lesions mimcking diabetic ophthalmoplegia. Clin Neurol Neurosurg, 1995;97(2):167-71.

5. Jacobson DM, Broste SK. Early progression of ophtalmoplegia in patients with ischemic oculomotor nerve palsies. Arch Ophthalmol, 1995;113(12):1535-7.

6. Trigler L, Siatkowski RM, Oster AS, Feuer WJ, betts CL, Glase JS, Schatz NJ, Farris

7. BK, Flynn HW. Retinopathy in patients with diabetic ophthalmoplegia.

8. Azaiez A, Marrakchi S, Rezgui H, Gharbi M, Kaoueche M, Jeddi A, Daghfous F, Ayed S. Les paralysies oculomotrices chez le diabétique. La Tunisie Médicale, 1996;74(11):489-91.

9. Greco D, Gambina F, Maggio F. Ophthalmoplegia in diabetes mellitus: aretrospective study. Acta Diabetol 2009; 46:23-26.

10. Lazzaroni F, Laffi GL, Galuppi V, Scorolli L. Paralysies des nerfs oculomoteurs au cours du diabète : étude rétrospective de 44 cas. Rev Neurol (Paris), 1993;149(10):571-3..

11. Jacobson DM, Mc Canna TD, Layde PM. Risk factors for ischemic ocular motor nerve palsies. Arch Ophthalmol, 1994;112(7):961.

12. Naghmi R and Subuki R. Diabetic oculomotor mononeuropathy: involvement of pupillomotor fibres with slow resolution. Horm Metabol Res, 1990;22:38-40.

13. Dreyfus PM, Hakim S, Adams RD. Diabetic ophthalmoplegia. Report of case, with

14. postmortem study and comments on vascular supply of human oculomotor nerve.

15. Arch Neurol Psychiatry 1957;77:337-349.

16. Hotamisligil GS. Inflammation and metabolic disorders. Nature 2006; 444: 860-7.

17. Kislinger T, Tanji N, Wendt T, Qu W, Lu Y, Ferran Jr.LJ, et al. Receptor for advanced glycation and products mediates inflammation and enhanced expressionof tissue factor in vasculature of diabetic apolipoproteïn ENULL MICE; ArteriosclerThromb Vasc Biol 2001; 21: 905-10.

18. Zorrilla E, Kozak GP. Ophthalmoplegia in diabetes mellitus. Ann Intern Med1967;67:968-976.

19. Watanabe K, Hargura R, Akanuma Y, Takasu T, Kajinuma H, Kuzuya N, Irie M. Characteristics of cranial nerve palsies in diabetic patients. Diabetes. Res Clin Pract, 1990;10(1):19-27. 
20. Lazzaroni F, Laffi GL, Galuppi V, Scorolli L. Paralysis of oculomotor nerves in diabetes mellitus. Aretrospective study of 44 cases. Rev Neurol 1993; 149: 571-3.

21. Hattori S, Utsunomiya K. Isolated oculomotor nerve palsy caused by mesencephalic hemorrhage. RinshoShimkeigaku, 1994; 34(10): 1021-5.

22. Orgawa K, Segawa F, Fujioka T, Kurihara T, Kinshita M. Unilateral external ophthalmoplegia caused by ipsilateral oculomotor nuclear lesion: analysis with diffusion weighted MRI. Risho-Shinkeigaku, 1994;34(9):914-7.

23. Boulton A; Rayaz AM; Arezzo JC, Sosenko JM. Diabetic somatic neuropathies.

24. Diabetes Care 2004; 27: 1458-1486. 